ARTICLE ORIGINAL / ORIGINAL ARTICLE

\title{
Le burn-out en réanimation : évaluation, prévention et dispositifs d'intervention
}

\author{
Burnout in intensive care medecine: evaluation, prevention and intervention
}

A. Fournier ${ }^{1,2} \cdot$ A. Laurent $t^{1,3 *}$

Reçu le 15 janvier 2020 ; accepté le 18 août 2020.

(c) SRLF 2020.

\section{Résumé}

Les professionnels de réanimation sont confrontés de façon répétitives et intenses à de nombreux facteurs de stress les rendant plus vulnérables au développement d'un burn-out. Le burn-out constitue le stade final d'une rupture d'adaptation suite à un déséquilibre entre les exigences professionnelles et les ressources de l'individu. II affecte directement la santé mentale et physique des professionnels et est associé à une diminution de la qualité et de la sécurité des soins et à une augmentation des départs des professionnels les plus expérimentés pour d'autres services. En réanimation de nombreuses études ont été menées sur les professionnels et les organisations lancent des appels à l'action pour lutter contre cette souffrance au travail. Le présent article fait un état des lieux de la prévalence du burn-out en réanimation. II présente également des dispositifs d'interventions individuelles et organisationnelles qui peuvent être utilisés pour prévenir et traiter le burn-out.

Mots-clés : burn-out, réanimation, stress, interventions, prévention

\section{Abstract}

Intensive care unit professionals are repetitively and intensely confronted with many stressful factors that make them more vulnerable to the development of burnout. Burnout is the final stage of a break in adaptation following an imbalance between professional demands and the individual's resources. It directly affects the mental and physical health of professionals and is associated with a decrease in the quality and safety of care and an increase in the departure of more experienced professionals for other units. Today, many studies in intensive care unitsare attempting to raise awareness of burnout among professionals and organizations are calling for action. This article presents an overview of the prevalence, assessment and prevention of burnout in intensive care units. It also presents individual and organizational interventions that can be used to prevent and treat burnout.

Keywords: burnout, intensive care units, stress, interventions, prevention

\section{Introduction}

Les professionnels de réanimation sont confrontés à des patients présentant des états pathologiques graves qui nécessitent des procédures diagnostiques et thérapeutiques complexes et qui exigent une importante coordination de moyens humains. La souffrance des patients associée à la détresse des familles et les décisions de fin de vie fréquentes participent à une intense charge émotionnelle pour les soignants $[1,2]$. Ce contexte de soin fait de la réanimation un terrain propice à l'émergence de stresseurs professionnels [3, 4]. Évalués par l'individu comme des situations affaiblissant ou excédant ses ressources [5] ces facteurs de stress constituent un terrain favorable à l'épuisement professionnel (ou burn-out) et particulièrement au sein des services de réanimation où le taux de prévalence du burn-out varie de 25 à $33 \%$ pour les infirmiers, $45 \%$ pour les méde-

\footnotetext{
*Alexandra Laurent

Laboratoire Psy-DREPI, Université de Bourgogne, Pôle AAFE, Esplanade Erasme, Dijon

$\nabla$ alexandra.laurent@u-bourgogne.fr

La liste complète des auteurs est disponible à la fin de l'article.
} 
cins et atteignant $71 \%$ chez les médecins travaillant en réanimation pédiatrique [6]. Le burn-out est associé à un risque accru de consommation de substances psychoactives, de pathologies cardiovasculaires, de douleurs musculo-squelettiques, de symptômes dépressifs et de façon moins systématique à des troubles du sommeil [7] . De plus, les conséquences du burn-out ne concernent pas seulement la santé des travailleurs. Selon plusieurs études, le burn-out entraîne des conséquences directes sur le travail : insatisfaction et désengagement au travail, diminution de la productivité et de l'engagement, taux élevé d'absentéisme, une augmentation du turnover [7-9], détérioration de la qualité et la sécurité des soins et diminution de la satisfaction des familles et des patients [10-13].

Selon Maslach et Jackson [14], le burn-out se définit comme une fatigue physique et émotionnelle qui amène l'individu à une conception négative de soi, à des attitudes négatives au travail et à une diminution progressive et importante de son implication au travail. Considéré comme un syndrome affectant les individus investissant intensément la relation d'aide dans le cadre de leur travail, Maslach et Jackson [14] émettent l'hypothèse que ce sont les conditions mêmes de la relation d'aide professionnelle, plutôt que des facteurs de personnalité, qui déterminent majoritairement la vulnérabilité au développement d'un burn-out. Ainsi, un « candidat » au burn-out, selon Delbrouck [15], serait celui qui manifesterait un enthousiasme idéaliste de sa profession.

Le burn-out ne survient pas subitement. II s'immisce dans un contexte de stress chronique lié à des facteurs organisationnels et relationnels au travail. Trois grandes dimensions permettent de décrire le processus du burnout : l'épuisement émotionnel, la déshumanisation de la relation à l'autre et la baisse de l'accomplissement de soi au travail [14]. L'ordre selon lequel sont présentées ces trois dimensions n'est pas indifférent. Le burn-out se développe progressivement, et ce, à l'insu du professionnel. Apparaît en premier lieu l'épuisement émotionnel. Autant physique que psychique, il se traduit par le sentiment d'être poussé au-delà de ses limites émotionnelles. Le soignant a l'impression d'être vidé, il ressent une fatigue affective au travail et une difficulté à être en relation avec les émotions du patient. À partir du moment où le soignant se sent submergé par des relations professionnelles trop coûteuses émotionnellement, le recours à une relation " déshumanisée " apparaît comme un moyen simple et immédiat de tenir à distance les émotions. La relation est alors marquée par un détachement et une sécheresse relationnelle s'apparentant au cynisme. Le soignant opère une véritable mise à distance psychologique ou comportementale du patient, de la famille et/ ou de ses collègues [16]. La déshumanisation constitue dans ce cadre une tentative de réponse à l'épuisement émotionnel et physique, mais en accroissant la distance psychologique entre l'individu et l'ensemble de sa pratique professionnelle, la déshumanisation ouvre la porte à la diminution de l'accomplissement personnel. Ainsi, peu à peu, l'intérêt professionnel disparaît derrière la technique et la routine. La motivation au travail diminue en même temps que le sentiment de bien faire son travail. Le soignant commence à douter de lui et de ses capacités professionnelles. II éprouve des difficultés importantes à être en contact avec les patients, les proches et son environnement professionnel. La diminution de l'accomplissement personnel entraîne un fort sentiment d'échec personnel, une diminution de l'estime de soi, du sentiment d'auto-efficacité et une frustration au travail.

L'épuisement professionnel se révèle donc être un véritable processus de dégradation du rapport de l'individu à son travail pouvant entraîner une symptomatologie dépressive sévère avec des risques suicidaires [17].

\section{Quelles évaluations du burn-out aujourd'hui?}

En 1981, Maslach et Jackson ont élaboré un instrument de mesure du burn-out à destination des professionnels travaillant dans la relation d'aide et de soins : le Maslach Burnout Inventory - Human Services Survey (MBI-HSS) [14]. Par la suite, plusieurs autres échelles ont été développées : le Burnout Measure [18], le Oldenburg Burnout Inventory [19], le Copenhagen Burnout Inventory [20], et la Shirom-Melamed Burnout Measure [21]. Cependant, le MBI-HSS reste actuellement l'échelle la plus utilisée pour mesurer l'épuisement professionnel dans les services de santé [22]'.

Le MBI-HSS a été l'objet de plusieurs études de validation successives qui ont toutes donné des résultats très satisfaisants [23, 24]. Cet outil se présente sous la forme d'un auto-questionnaire constitué de 22 items, évaluant les trois dimensions du burn-out : l'épuisement émotionnel (EE) (neuf items), la dépersonnalisation (DP) (cinq items) et l'accomplissement personnel (AP) (huit items). La cotation du MBI-HSS en sept points $(0=$ jamais ; $6=$ tous les jours) ne permet pas de calculer un score global de burn-out, mais permet d'obtenir un degré de sévérité (bas, modéré, élevé) pour chacune des dimensions. Ce degré de sévérité est défini par des seuils moyens obtenus dans un ensemble de profession d'aide et de soin : EE (burn-out bas : score < 17, burn-out modéré : 17 à 26 , burn-out élevé : > 26) ; DP (burn-out bas : $<7$, burn-out modéré : 7 à 12, burn-out élevé : $>12$ ) ; AP (burn-out élevé : $<32$, burn-out modéré : 32 à 38 , burn-out bas : > 38) [14]. Bien que le MBI soit l'outil de référence

${ }^{1}$ Le MBI possède des droits d'utilisation. Il est possible d'acheter ces droits avec une mise à disposition d'une traduction française à l'adresse suivante : https://www.mindgarden.com/315-mbi-human-services-surveymedical-personnel. srlf 
pour l'estimation du burn-out, sa validité et sa fiabilité font souvent l'objet de débats. Plusieurs chercheurs ont montré que les dimensions essentielles du burn-out sont les dimensions EE et DP [25-28]. Certains préconisent uniquement l'utilisation de ces deux dimensions [29]. Plus récemment, des auteurs proposent un modèle à deux facteurs, consistant en un facteur d'épuisement, combinant les dimensions EE et DP, et un facteur distinct représenté par la dimension AP [30].

Soulignons enfin que ces dimensions ne sont pas les éléments d'une triade diagnostique ou d'un ensemble de critères relevant d'une classification nosographique. II n'existe pas de diagnostic de burn-out en tant que tel. Au regard de la proximité du burn-out avec les symptômes de dépression, d'anxiété et de stress post-traumatique, certains chercheurs conseillent d'ailleurs l'utilisation complémentaire d'échelles de dépression, d'anxiété et de stress post-traumatique afin de faciliter l'évaluation clinique et l'interprétation des résultats obtenus [31, 32].

Au-delà du diagnostic, le burn-out est avant tout un processus mettant en exergue la souffrance au travail. Dans ce sens, Daloz [33] stipule que le burn-out n'est pas vraiment spécifique en tant que tableau clinique, et qu'il serait plutôt à considérer comme un signal d'alarme que les soignants manifesteraient pour ne pas « mourir d'épuisement ». Ainsi, l'entité burn-out permettrait de conceptualiser cette confrontation à un risque psychique majeur face à des attentes, des idéaux professionnels insatisfaits. Cette terminologie a l'avantage aujourd'hui d'opérer un effet mobilisateur autour d'un malaise mettant en exergue les sources de dysfonctionnement des équipes et des institutions et rendant indispensable la mise en place de dispositifs de prévention et d'accompagnement.

\section{Prévention et dépistage}

Aujourd'hui, un grand nombre de facteurs de stress ont été identifiés comme étant corrélés au burn-out. Ces facteurs concernent à la fois des caractéristiques personnelles (e.g., le genre, l'âge, la durée d'expérience professionnelle dans les services de réanimation, le statut marital, les croyances), des facteurs organisationnels (e.g., le nombre de garde, le travail de nuit, le nombre de jours de travail consécutifs, l'environnement de travail), la qualité des relations de travail (e.g., les conflits interpersonnels, l'animosité, la méfiance, le manque de communication) et des questions de fin de vie (e.g., les soins d'un patient mourant, assister aux décisions d'arrêt de traitement $[6,10])$. Si l'identification des facteurs de stress est important en termes de prévention du burnout, il s'avère que les outils permettant l'identification des facteurs de stress en réanimation sont pour la majorité des échelles non validées ou des échelles « génériques » non spécifiques à l'environnement de travail en santé (e.g., Job Content Questionnaire (JCQ) [34] ; EffortReward-Imbalance (ERI) [35]) qui ne prennent pas en compte les facteurs de stress les plus spécifiques à la réanimation tels que la gestion du risque vital/urgence, et les dilemmes moraux/éthiques [36]. Les conséquences de ce manque d'outils valides et spécifiques sont la difficulté d'identifier les facteurs de stress les plus pertinents liés à la souffrance au travail, et de mettre en place des mesures correctrices adaptées.

La question du dépistage du burn-out dans les services de réanimation pour prévenir et réduire le burnout a fait l'objet d'avis divergents [32, 37, 38]. Selon Papazian et ses collègues, le burn-out devrait être dépisté régulièrement pour faciliter l'identification des facteurs impactant le plus le bien-être des soignants, faciliter l'identification des soignants qui nécessite un soutien, et assurer et promouvoir des stratégies visant à améliorer l'environnement de travail. Mealer et Moss sont eux plus mitigés. La mesure du burn-out n'étant pas un outil diagnostic, son utilisation risque de ne pas être adapté. Par ailleurs, compte tenu de l'importance d'autres troubles psychologiques chez les soignants en réanimation (anxiété, dépression, état de stress posttraumatique), le dépistage du burn-out à lui seul n'est pas suffisant. Une autre dimension importante est soulignée, le burn-out est actuellement fortement stigmatisé et un dépistage systématique pourrait être interprété comme une surveillance réglementaire supplémentaire et ainsi aggraver le burn-out [37]. Par conséquent, des approches éducatives sur ce qu'est l'épuisement professionnel et des interventions organisationnelles et individuelles doivent être associées au dépistage [32].

\section{Dispositifs d'interventions individuelles et organisationnelles}

Au sein de la littérature les interventions se répartissent selon deux grandes modalités : des interventions dites individuelles ayant une action sur les facteurs/ressources internes aux soignants et des interventions dites organisationnelles basées sur les facteurs externes aux soignants.

Au niveau individuel, van Mol et ses collègues listent un ensemble de stratégies préventives individuelles pour réduire notamment le burn-out dans les services de réanimation [39]. Parmi ces interventions, il est proposé des interventions centrées sur la pratique (mises en place de séminaires et des programmes éducatifs centrés sur la pratique, améliorer les éléments de soins axés sur la famille et les capacités de communication, proposer des exercices de relaxation et de pleine conscience) et des interventions centrées sur les caractéristiques individuelles (proposer des programmes de développement 
de l'intelligence émotionnelle, agir sur la personnalité et les stratégies d'adaptation des soignants, possibilité de rencontrer un psychologue extérieur au service). Moss et ses collègues [6] recensent également un ensemble de stratégies clefs pour réguler le stress et prévenir le burn-out : apprendre à reconnaître les facteurs de risque du burn-out et savoir demander de l'aide, mettre en place des comportements d'auto-soin (i.e., les décisions et actions que la personne met en place pour améliorer sa santé, prévenir le développement de maladies, limiter la maladie et rétablir sa santé), établir un équilibre entre le travail et la vie personnelle, avoir recours à des formations en gestion du temps, des groupes de soutien et la mise en place de techniques de gestion de l'état de stress (i.e., groupes de soutien, programmes de réduction du stress par la pleine conscience (Mindfulness-based stress reduction (MBSR) [40]), thérapie cognitivo-comportementale).

Pour répondre au contexte spécifique qu'est la réanimation, des équipes de recherche ont également élaboré des nouveaux programmes pour aider les soignants à faire face à l'adversité. Mealer et ses collègues ont élaboré un programme de formation à la résilience chez les infirmiers en réanimation [41]. La résilience suppose de s'adapter aux situations difficiles en mettant à contribution des modalités de protection relevant des ressources internes et externes au sujet afin de maintenir un équilibre psychologique malgré les situations difficiles [42]. Ce programme de 12 semaines incluait des ateliers éducatifs, des thérapies d'exposition écrite, des pratiques de pleine conscience (MBSR), des exercices physiques cardiovasculaires et des séances de conseils déclenchées par des évènements dans le milieu professionnel. Ce dispositif a permis de diminuer les scores de dépression, de stress post-traumatique et a permis d'améliorer les scores de résilience. Les résultats obtenus restent prometteurs dans le cadre de la souffrance au travail et cette étude montre la faisabilité de ce type d'intervention chez des infirmiers en réanimation. Une équipe française s'est centrée sur les techniques de simulation pour aider les infirmiers à s'adapter aux situations stressantes rencontrées en réanimation (stress en lien avec la prise en charge des patients, l'organisation du travail, et les conditions de travail) [43]. Les objectifs des séances de simulation étaient d'améliorer la qualité et la sécurité des soins, de mettre à jour la théorie nécessaire au raisonnement clinique et à la prise de décision, et d'acquérir/développer les compétences des infirmières en réanimation. Six mois après ce programme d'intervention, le stress professionnel perçu (mesuré par le JCQ), le taux d'absentéisme et de turnover avaient diminué, et la satisfaction au travail augmenté [43]. Il est important de noter que renforcer la résilience ou proposer uniquement des interventions

Tableau 1 - Proposition d'interventions centrées sur l'individu pour diminuer le burn-out et promouvoir le bien-être au travail dans les services de réanimation à partir des revues de la littérature de Moss et al., 2016 ; Shanafelt et al., 2016 ; van Mol et al., 2015 ; Murali et al., 2018

\section{Cibles d'interventions}

Établir un équilibre entre le travail et la vie personnelle

Programmes et séminaires éducatifs

Favoriser la verbalisation des émotions

Amélioration des compétences personnelles

Agir sur la personnalité et les stratégies d'adaptation

Exercices de relaxation
Exemple d'interventions

Savoir prendre de la distance avec le travail, prendre des vacances $[6,46]$

Séances de soutien animés pour réduire l'impact émotionnel des soins palliatifs

[47] ; Séminaires éducatifs [48] ; Apprendre à reconnaître les facteurs de risque au développement d'un burn-out [6] ; Réaliser une formation Balint [49]

Possibilité de consulter un psychologue pour les soignants [6, 50]; Programme basé sur de la réflexion et partage d'expérience [51,52]; Programme de soutien et informations

[53] ; Réaliser des séances de débriefing [54] ; Réaliser des interventions de conseils [55, 56] ; Réaliser des ateliers d'écriture réflexive [57]

Programmes éducatifs sur l'intelligence émotionnelle [10, 58, 59] ; Apprendre à gérer son temps [6]

Apprendre aux individus à maintenir leur santé émotionnelle et physique [6] ; Programme d'entraîement à la résilience [41] ; Proposer des ateliers d'auto-soins [60, 61]

Pratiquer du yoga [62] ; Avoir un passe-temps en dehors du travail [6] ; Pratiquer la pleine conscience (MBSR) [6, 51, 63] ; Pratiquer la méditation [64] ; Réaliser des programmes de gestion de l'état de stress $[51,65,66]$ 
centrées sur les individus sont des stratégies qui négligent les facteurs organisationnels qui sont les principaux moteurs du burn-out des soignants en réanimation [44]. Ces interventions confortent également un discours institutionnel interprétant l'épuisement professionnel comment émanant de la responsabilité exclusive de l'individu [44]. Ainsi, les interventions organisationnelles restent essentielles pour promouvoir le bien-être au travail et lutter contre la souffrance au travail. Ces stratégies peuvent être regroupées en onze grandes catégories [6, 39, 44, 45] (Tableau 1 et tableau 2) :

1. améliorer l'environnement de travail ;

2. changer la composition des équipes soignantes ;
3. développer un travail d'équipe pour augmenter la cohésion et permettre des décisions efficaces ;

4. éviter la monotonie du travail ;

5. améliorer les capacités de communication ;

6. favoriser les discussions d'éthique ;

7. promouvoir le pouvoir d'un leadership ;

8. reconnaître le travail fourni par les soignants ;

9. promouvoir l'équilibre entre la vie professionnelle et personnelle ;

10.fournir les ressources pour promouvoir la résilience et l'auto-soin ;

11. établir une culture organisationnelle et financer la recherche en sciences des organisations.

\section{Tableau 2 - Proposition d'interventions centrées sur l'organisation pour diminuer le burn-out et promouvoir le bien- être au travail dans les services de réanimation à partir des revues de la littérature de Moss et al., 2016 ; Shanafelt et al., 2016 ; van Mol et al., 2015 ; Murali et al., 2018}

\begin{tabular}{|c|c|}
\hline Cibles d'interventions & Exemple d'interventions \\
\hline $\begin{array}{l}\text { Amélioration de } \\
\text { l'environnement de travail }\end{array}$ & Prévenir/Gérer les conflits $[63,67,68]$; Introduction d'heures de visite sans restriction [63] \\
\hline $\begin{array}{l}\text { Changement de la } \\
\text { composition de l'équipe } \\
\text { soignantes }\end{array}$ & $\begin{array}{l}\text { Taille et composition appropriées de l'équipe [6] ; Introduire des assistants médicaux pour } \\
\text { accompagner les médecins (Programme APEX) [69] }\end{array}$ \\
\hline $\begin{array}{l}\text { Développer le travail } \\
\text { d'équipe }\end{array}$ & $\begin{array}{l}\text { Organiser des réunions pour faciliter les prises de décisions efficaces [6] ; Mettre en place } \\
\text { des salles de réunion favorisant les échanges [44] }\end{array}$ \\
\hline Éviter la monotonie & Effectuer des roulements dans les tâches professionnelles [70] \\
\hline $\begin{array}{l}\text { Améliorer les capacités de } \\
\text { communication }\end{array}$ & $\begin{array}{l}\text { Réunion entre l'équipe soignante et le patient et/ou sa famille pour discuter des objectifs } \\
\text { de traitement }[6,63] \text {; Heures de temps de travail protégées pour rencontrer les collègues } \\
\text { et discuter des expériences professionnelles }[44,51] \text {; Réunion entre les leaders des } \\
\text { différentes équipes pour faciliter la coopération }[44] \text {; Discussion avec les dirigeants de } \\
\text { l'institution [44] ; Possibilité de consulter un psychologue pour les soignants }[6,50]\end{array}$ \\
\hline $\begin{array}{l}\text { Favoriser les discussions } \\
\text { d'éthique }\end{array}$ & $\begin{array}{l}\text { Une discussion éthique dans chaque dossier médical du patient ou dans le cadre des } \\
\text { séances d'évaluation éthique [63]; Discussions interdisciplinaires qui favorisent les } \\
\text { délibérations éthiques en équipe et créent un environnement de confiance [6] }\end{array}$ \\
\hline $\begin{array}{l}\text { Promouvoir le pouvoir d'un } \\
\text { leadership }\end{array}$ & {$[6] ;[44] ;[71]$} \\
\hline $\begin{array}{l}\text { Reconnaissance } \\
\text { significative du travail }\end{array}$ & [6] \\
\hline $\begin{array}{l}\text { Promouvoir l'équilibre entre } \\
\text { la vie professionnelle et } \\
\text { personnelle }\end{array}$ & $\begin{array}{l}\text { Permettre aux médecins d'adapter leur emploi du temps en fonction de leurs obligations } \\
\text { personnelles [44] ; Repos de fin de semaine [72] ; Favoriser un modèle composé de sept } \\
\text { jours de travail consécutifs avec un autre médecin pour les nuits [73] ; Restreindre les } \\
\text { heures de travail par semaine [74] }\end{array}$ \\
\hline $\begin{array}{l}\text { Fournir les ressources pour } \\
\text { promouvoir la résilience et } \\
\text { l'auto-soin }\end{array}$ & $\begin{array}{l}\text { Fournir aux soignants les ressources qui permettent de mettre en œuvre plus facilement } \\
\text { des stratégies individuelles pour prévenir le burn-out, faire face à la détresse et } \\
\text { promouvoir le bien-être [44] }\end{array}$ \\
\hline $\begin{array}{l}\text { Établir une culture } \\
\text { organisationnelle et } \\
\text { financer la science } \\
\text { organisationnelle }\end{array}$ & $\begin{array}{l}\text { Créer une culture organisationnelle qui empêche la stigmatisation du burnout }[32,75] \text {; } \\
\text { Faciliter et financer la recherche en science des organisations }[44]\end{array}$ \\
\hline
\end{tabular}




\section{Conclusion}

L'hôpital est un lieu de souffrance où les professionnels se confrontent aux émotions les plus intenses. Ils côtoient les situations les plus extrêmes au sein d'une organisation, ce qui peut épuiser les ressources individuelles et collectives et aller à l'encontre des valeurs morales et éthiques des professionnels. Cette souffrance, les professionnels de santé en sont les témoins, mais également les victimes et le burn-out l'illustre particulièrement. Le burn-out a aujourd'hui l'avantage de servir de grille de lecture pour décrire des comportements spécifiques de soignants confrontés dans leur quotidien professionnel à la répétition de situation de stress et à la violence des émotions. Ainsi, prendre en compte le burn-out en tant que processus, et non comme une entité diagnostique figée, nous permet de relancer la recherche et de développer des études longitudinales et qualitatives pour mieux comprendre les processus qui amènent le soignant à cet état d'épuisement. Les dispositifs d'accompagnement prennent aujourd'hui appui sur le concept de résilience. Ce concept a l'avantage d'appréhender l'accompagnement à la fois en lien avec les ressources internes du sujet, mais également les ressources externes constituées par son environnement. Ainsi, la prise en charge de l'épuisement professionnel doit être élaborée selon une approche multimodale à la fois centrée sur l'individu, mais également en lien avec une entité plus vaste où les problématiques liées aux patients, aux familles, au service, à l'institution et aux politiques de soins doivent être travaillées.

\section{Conflits d'intérêts}

Alicia Fournier et Alexandra Laurent déclarent ne pas avoir de conflit d'intérêts.

\author{
Affiliations \\ ${ }^{1}$ Laboratoire Psy-DREPI \\ Université de Bourgogne Franche-Comté \\ Dijon, France \\ ${ }^{2}$ Laboratoire de psychologie \\ Université Bourgogne Franche-Comté \\ Besançon, France \\ ${ }^{3}$ Réanimation Chirurgicale \\ CHU Dijon, France
}

\section{Références}

1. Flannery L, Ramjan LM, Peters K (2016) End-of-life decisions in the Intensive Care Unit (ICU) - Exploring the experiences of ICU nurses and doctors - A critical literature review. Aust Crit Care 29:97-103. DOI : 10.1016/j.aucc.2015.07.004

2. Laurent $A$, Bonnet $M, A n s e l D$ (2015) Les professionnels de réanimation à l'épreuve de la relation avec le patient. L'Évolution Psychiatr 80: 809-820 DOI : 10.1016/j.evopsy.2014.12.007
3. Donchin Y, Seagull FJ (2002) The hostile environment of the intensive care unit. Curr Opin Crit Care 8:316-320. DOI : 10.1097/00075198200208000-00008

4. Laurent A, Capellier G (2018) Stress professionnel en médecine intensive réanimation, de quoi parle-t-on? Méd Intensive Réa 27:75-79. DOI : 10.3166/rea-2018-0010

5. Lazarus RS, Folkman S, (1984) Stress, appraisal, and coping. Springer, New York, $456 p$

6. Moss M, Good VS, Gozal D, et al (2016) An Official Critical Care Societies Collaborative Statement-Burnout Syndrome in Critical Care Health-care Professionals: A Call for Action. Chest 150:17-26. DOI : 10.1016/j.chest.2016.02.649

7. Salvagioni DAJ, Melanda FN, Mesas AE, et al (2017) Physical, psychological and occupational consequences of job burnout: $A$ systematic review of prospective studies. PLoS One 12:e0185781. DOI : 10.1371/journal.pone.0185781

8. Brindley PG (2017) Psychological burnout and the intensive care practitioner: A practical and candid review for those who care. J Intensive Care Soc 18:270-275. DOI : 10.1177/1751143717713088

9. Ahola K, Kivimäki M, Honkonen T, et al (2008) Occupational burnout and medically certified sickness absence: a population-based study of Finnish employees. J Psychosom Res 64:185-193. DOI : 10.1016/j. jpsychores.2007.06.022

10. Pastores SM, Kvetan V, Coopersmith CM, et al (2019) Workforce, Workload, and Burnout Among Intensivists and Advanced Practice Providers: A Narrative Review. Crit Care Med 47:550-557. DOI : 10.1097/ CCM.0000000000003637

11. West CP, Tan AD, Habermann TM, et al (2009) Association of resident fatigue and distress with perceived medical errors. JAMA 302:1294-1300. DOI : 10.1001/jama.2009.1389

12. Welp A, Meier LL, Manser T (2016) The interplay between teamwork, clinicians' emotional exhaustion, and clinician-rated patient safety: a longitudinal study. Crit Care 20: 110. DOI : 10.1186/s13054-016-1282-9

13. Laurent A, Aubert L, Chahraoui K, et al (2014) Error in intensive care: psychological repercussions and defense mechanisms among health professionals. Crit Care Med 42:2370-2378. DOI : 10.1097/ CCM.0000000000000508

14. Maslach C, Jackson SE, (1981) Maslach Burnout Inventory. Consulting Psychologists Press, Palo Alto, CA

15. Delbrouck M (2010) « Je suis épuisé(e) par ma charge de travail. Que puis-je y faire ? Le burn-out ou la souffrance des soignants. Causes spécifiques du syndrome d'épuisement professionnel du soignant », Imaginaire \& Inconscient, vol. 25, no. 1, pp. 157-166.

16. Canouï P,Mauranges A (1998) Le syndrome d'épuisement professionnel des soignants. De l'analyse du burn out aux réponses. Elsevier Masson, Paris, France

17. Lesage F-X, Berjot S, Altintas E, Paty B (2013) Burnout among occupational physicians: a threat to occupational health systems?-A nationwide cross-sectional survey. Ann Occup Hyg 57:913-919. DOI : 10.1093/annhyg/met013

18. Pines A, Aronson E, Kafry D, (1981) Burnout: From tedium to personal growth. Free Press., New York

19. Halbesleben JRB, Demerouti E (2005) The construct validity of an alternative measure of burnout: Investigating the English translation siff 
of the Oldenburg Burnout Inventory. Work \& Stress 19:208-220. DOI : 10.1080/02678370500340728

20. Kristensen TS, Borritz M, Villadsen E, Christensen KB (2005) The Copenhagen Burnout Inventory: A new tool for the assessment of burnout. Work \& Stress 19:192-207. DOI : 10.1080/02678370500297720

21. Shirom A, Melamed S (2006) A comparison of the construct validity of two burnout measures in two groups of professionals. Int J Stress Manag 13: 176-200. DOI : 10.1037/1072-5245.13.2.176

22. Lheureux F, Truchot D, Borteyrou X, Rascle N (2017) The maslach burnout inventory - Human services survey (mbi-hss): factor structure, wording effect and psychometric qualities of known problematic items. Le travail humain Vol. 80:161-186

23. Maslach C, Schaufeli WB, Leiter MP (2001) Job burnout. Annu Rev Psychol 52:397-422. DOI : 10.1146/annurev.psych.52.1.397

24. Bakker AB, Demerouti E, Verbeke W (2004) Using the job demandsresources model to predict burnout and performance. Human Resource Management 43:83-104. DOI : 10.1002/hrm.20004

25. Kanste O, Miettunen J, Kyngäs H (2006) Factor structure of the Maslach Burnout Inventory among Finnish nursing staff. Nurs Health Sci 8:201-207. DOI : 10.1111/j.1442-2018.2006.00283.x

26. Demerouti E, Bakker AB, Nachreiner F, Schaufeli WB (2001) The job demands-resources model of burnout. J Appl Psychol 86:499-512

27. Demerouti E, Mostert K, Bakker AB (2010) Burnout and work engagement: a thorough investigation of the independency of both constructs. J Occup Health Psychol 15:209-222. DOI : 10.1037/ a0019408

28. Peterson U, Demerouti E, Bergström G, et al (2008) Burnout and physical and mental health among Swedish healthcare workers. J Adv Nurs 62:84-95. DOI : 10.1111/j.1365-2648.2007.04580.x

29. Nori P, Bartash R, Cowman K, et al (2019) Is Burnout Infectious? Understanding Drivers of Burnout and Job Satisfaction Among Academic Infectious Diseases Physicians. Open Forum Infect Dis 6:ofzO92. DOI : 10.1093/ofid/ofz092

30. de Beer LT, Bianchi R (2019) Confirmatory Factor Analysis of the Maslach Burnout Inventory. Eur J Psychol Assess 35: 217-224. DOI : 10.1027/1015-5759/a000392

31. Bianchi R, Schonfeld IS, Laurent E (2015) Burnout-depression overlap: a review. Clin Psychol Rev 36:28-41. DOI : 10.1016/j.cpr.2015.01.004

32. Mealer M, Moss M (2018) Should all ICU clinicians regularly be tested for burnout? We are not sure : Conceptual considerations that precede screening for burnout syndrome in ICU clinicians. Intensive Care Med 44:687-689. DOI : 10.1007/s00134-018-5169-x

33. Daloz L (2007) Epuisement professionnel et blessures de l'idéal. Réflexions cliniques sur la désillusion des soignants et la formation initiale. Pédagogie Médicale 8:82-90. DOI : 10.1051/pmed:2007003

34. Karasek R, Brisson C, Kawakami N, et al (1998) The Job Content Questionnaire (JCQ): an instrument for internationally comparative assessments of psychosocial job characteristics. J Occup Health Psychol 3:322-355. DOI : 10.1037//1076-8998.3.4.322

35. Siegrist J, Starke D, Chandola T, et al (2004) The measurement of effort-reward imbalance at work: European comparisons. Soc Sci Med 58:1483-1499. DOI : 10.1016/S0277-9536(03)00351-4

36. Laurent A, Lheureux F, Genet M, et al (2020) Scales Used to Measure Job Stressors in Intensive Care Units: Are They Relevant and
Reliable? A Systematic Review. Front Psychol 11:245. DOI : 10.3389/ fpsyg.2020.00245

37. Ricou B, Zampieri FG, Brown SM (2018) Should all ICU clinicians regularly be tested for burnout? No. Intensive Care Med 44:684-686. DOI : 10.1007/s00134-018-5139-3

38. Papazian L, Sylvestre A, Herridge M (2018) Should all ICU clinicians regularly be tested for burnout? Yes. Intensive Care Med 44:681-683. DOI : 10.1007/s00134-018-5094-z

39. van Mol MMC, Kompanje EJO, Benoit DD, et al (2015) The Prevalence of Compassion Fatigue and Burnout among Healthcare Professionals in Intensive Care Units: A Systematic Review. PLoS One 10:e0136955. DOI : 10.1371/journal.pone.0136955

40. Kabat-Zinn J (1982) An outpatient program in behavioral medicine for chronic pain patients based on the practice of mindfulness meditation: theoretical considerations and preliminary results. Gen Hosp Psychiatry 4:33-47. DOI : 10.1016/0163-8343(82)90026-3

41. Mealer M, Conrad D, Evans J, et al (2014) Feasibility and acceptability of a resilience training program for intensive care unit nurses. Am J Crit Care 23:e97-105. DOI : 10.4037/ajcc2014747

42. Herrman H, Stewart DE, Diaz-Granados N, et al (2011) What is resilience? Can J Psychiatry 56:258-265. DOI : 10.1177/070674371105600504

43. El Khamali R, Mouaci A, Valera S, et al (2018) Effects of a Multimodal Program Including Simulation on Job Strain Among Nurses Working in Intensive Care Units: A Randomized Clinical Trial. JAMA 320:1988-1997. DOI : 10.1001/jama.2018.14284

44. Shanafelt TD, Mungo M, Schmitgen J, et al (2016) Longitudinal Study Evaluating the Association Between Physician Burnout and Changes in Professional Work Effort. Mayo Clin Proc 91:422-431. DOI : 10.1016/j.mayocp.2016.02.001

45. Murali K, Makker V, Lynch J, Banerjee S (2018) From Burnout to Resilience: An Update for Oncologists. Am Soc Clin Oncol Educ Book 38:862-872. DOI : 10.1200/EDBK_201023

46. Liu K, You L-M, Chen S-X, et al (2012) The relationship between hospital work environment and nurse outcomes in Guangdong, China: a nurse questionnaire survey. J Clin Nurs 21:1476-1485. DOI : 10.1111/j.1365-2702.2011.03991.x

47. Eagle S, Creel A, Alexandrov A (2012) The effect of facilitated peer support sessions on burnout and grief management among health care providers in pediatric intensive care units: a pilot study. J Palliat Med 15:1178-1180. DOI : 10.1089/.jpm.2012.0231

48. Meadors P, Lamson A (2008) Compassion fatigue and secondary traumatization: provider self care on intensive care units for children. J Pediatr Health Care 22:24-34. DOI : 10.1016/j.pedhc.2007.01.006

49. Ghetti C, Chang J, Gosman G (2009) Burnout, psychological skills, and empathy: balint training in obstetrics and gynecology residents. J Grad Med Educ 1:231-235. DOI : 10.4300/JGME-D-09-00049.1

50.Lederer W, Kinzl JF, Traweger C, et al (2008) Fully developed burnout and burnout risk in intensive care personnel at a university hospital. Anaesth Intensive Care 36:208-213. DOI : 10.1177/0310057X0803600211

51. West CP, Dyrbye LN, Rabatin JT, et al (2014) Intervention to promote physician well-being, job satisfaction, and professionalism: a randomized clinical trial. JAMA Intern Med 174:527-533. DOI : 10.1001/ jamainternmed.2013.14387 
52. Ripp JA, Fallar R, Korenstein D (2016) A Randomized Controlled Trial to Decrease Job Burnout in First-Year Internal Medicine Residents Using a Facilitated Discussion Group Intervention. J Grad Med Educ 8:256-259. DOI : 10.4300/JGME-D-15-00120.1

53. Loiselle CG, Gélinas C, Cassoff J, et al (2012) A pre-post evaluation of the Adler/Sheiner Programme (ASP): a nursing informational programme to support families and nurses in an intensive care unit (ICU). Intensive Crit Care Nurs 28:32-40. DOI : 10.1016/j.iccn.2011.11.006

54. Gunasingam N, Burns K, Edwards J, et al (2015) Reducing stress and burnout in junior doctors: the impact of debriefing sessions. Postgrad Med J 91:182-187. DOI : 10.1136/postgradmedj-2014-132847

55. Rø KEl, Gude T, Tyssen R, Aasland OG (2008) Counselling for burnout in Norwegian doctors: one year cohort study. BMJ 337:a2004. DOI : 10.1136/bmj.a2004

56. Isaksson Ro KE, Tyssen R, Hoffart A, et al (2010) A three-year cohort study of the relationships between coping, job stress and burnout after a counselling intervention for help-seeking physicians. BMC Public Health 10:213. DOI : 10.1186/1471-2458-10-213

57. Winkel AF, Hermann N, Graham MJ, Ratan RB (2010) No time to think: making room for reflection in obstetrics and gynecology residency. J Grad Med Educ 2:610-615. DOI : 10.4300/JGME-D-10-00019.1

58. Nooryan K, Gasparyan K, Sharif F, Zoladl M (2012) Controlling anxiety in physicians and nurses working in intensive care units using emotional intelligence items as an anxiety management tool in Iran. Int J Gen Med 5:5-10. DOI : 10.2147/IJGM.S25850

59. Nooryan K, Gasparyan K, Sharif F, Zoladl M (2011) The effect of teaching emotional intelligence (EI) items on job related stress in physicians and nurses working in ICU wards in hospitals, Yerevan, Armenia. Int J Collab Res Intern Med Public Heal 3: 704-713

60. Martins AE, Davenport MC, Del Valle MP, et al (2011) Impact of a brief intervention on the burnout levels of pediatric residents. J Pediatr (Rio J) 87:493-498. DOI : 10.2223/JPED.2127

61. Weight CJ, Sellon JL, Lessard-Anderson CR, et al (2013) Physical activity, quality of life, and burnout among physician trainees: the effect of a team-based, incentivized exercise program. Mayo Clin Proc 88:1435-1442. DOI : 10.1016/j.mayocp.2013.09.010

62. Mehrabi T, Azadi F, Pahlavanzadeh S, Meghdadi N (2012) The effect of yoga on coping strategies among intensive care unit nurses. Iran J Nurs Midwifery Res 17:421-424

63. Quenot JP, Rigaud JP, Prin S, et al (2012) Suffering among carers working in critical care can be reduced by an intensive communication strategy on end-of-life practices. Intensive Care Med 38:55-61. DOI : 10.1007/s00134-011-2413-z

64. Oman D, Hedberg J, Thoresen CE (2006) Passage meditation reduces perceived stress in health professionals: a randomized, controlled trial. J Consult Clin Psychol 74:714-719. DOI : 10.1037/0022006X.74.4.714

65. Rowe MM (2006) Four-year longitudinal study of behavioral changes in coping with stress. Am J Health Behav 30:602-612. DOI : 10.5555/ ajhb.2006.30.6.602

66. Krasner MS, Epstein RM, Beckman H, et al (2009) Association of an educational program in mindful communication with burnout, empathy, and attitudes among primary care physicians. JAMA 302:1284-1293. DOI : 10.1001/jama.2009.1384
67. Azoulay E, Timsit J-F, Sprung CL, et al (2009) Prevalence and factors of intensive care unit conflicts: the conflicus study. Am J Respir Crit Care Med 180:853-860. DOI : 10.1164/rccm.200810-16140C

68. Chlan LL, RN, PhD, FAAN Burnout Syndrome Among Critical Care Professionals: A Cause for Alarm. https://www.reliasmedia.com/ articles/64302-burnout-syndrome-among-critical-care-professionals-acause-for-alarm?v=preview. Accessed 15 Oct 2020

69. Wright AA, Katz IT (2018) Beyond Burnout - Redesigning Care to Restore Meaning and Sanity for Physicians. NEngl J Med 378:309-311. DOI : 10.1056/NEJMp1716845

70. Bellieni CV, Righetti P, Ciampa R, et al (2012) Assessing burnout among neonatologists. J Matern Fetal Neonatal Med 25:2130-2134. DOI : 10.3109/14767058.2012.666590

71. Goetz K, Beutel S, Mueller G, et al (2012) Work-related behaviour and experience patterns of nurses. International Nursing Review 59:88-93. DOI : 10.1111/j.1466-7657.2011.00921.x

72. Ali NA, Hammersley J, Hoffmann SP, et al (2011) Continuity of care in intensive care units: a cluster-randomized trial of intensivist staffing. Am J Respir Crit Care Med 184:803-808. DOI : 10.1164/rccm.20110305550C

73. Garland A, Roberts D, Graff L (2012) Twenty-four-hour intensivist presence: a pilot study of effects on intensive care unit patients, families, doctors, and nurses. Am J Respir Crit Care Med 185:738-743. DOI : 10.1164/rccm.201109-17340C

74. West CP, Dyrbye LN, Erwin PJ, Shanafelt TD (2016) Interventions to prevent and reduce physician burnout: a systematic review and metaanalysis. Lancet 388:2272-2281. DOI : 10.1016/S0140-6736(16)31279-X 75. Mealer M (2016) Burnout Syndrome in the Intensive Care Unit. Future Directions for Research. Ann Am Thorac Soc 13:997-998. DOI : 10.1513/AnnalsATS.201604-280ED siff 